\title{
Self-Nulling Eddy Current Probe for Surface and Subsurface Flaw Detection
}

\author{
B. Wincheski ${ }^{1}$, J. P. Fulton ${ }^{1}$, S. Nath ${ }^{1}$, M. Namkung ${ }^{2}$, and J. W. Simpson ${ }^{3}$
}

An eddy current probe which provides a null-signal in the presence of unflawed material without the need for any balancing circuitry has been developed at NASA Langley Research Center [1]. Such a unique capability of the probe reduces set-up time, eliminates tester configuration errors, and decreases instrumentation requirements. The probe is highly sensitive to surface breaking fatigue cracks, and shows excellent resolution for the measurement of material thickness, including material loss due to corrosion damage. The presence of flaws in the material under test causes an increase in the extremely stable and reproducible output voltage of the probe. The design of the probe and some examples illustrating its flaw detection capabilities are presented.

\section{Probe Design}

The self-nulling eddy current probe is a driver-pickup type eddy current probe which has a ferromagnetic shield inserted between the drive and sensor coils. The ferromagnetic material focuses the flux of the probe into a tight cylinder about the outer diameter of the pickup coil. In addition, the direct mutual inductance between the drive and pickup coils is eliminated due to the skin depth attenuation of the fields as they penetrate inward from the drive to pickup coils [2]. Therefore, in the presence of an unflawed sample, there is no flux linkage through the pickup coil. Measurements are made with an $\mathrm{AC}$ voltmeter connected across the leads of the pickup coil. The potential across the coil is proportional to the time rate of change of the flux linkage [3], so that for an unflawed sample, with no flux linkage, a zero, or null voltage is measured. A schematic diagram of the probe configuration is given in Fig. 1. The magnetizing coil contains approximately 100 turns of AWG 28 copper wire and the pickup coil contains approximately 600 turns or AWG 38 copper wire.

The material for the shield was chosen so as to minimize the flux linkage through the pickup coil while maintaing a large field density at the outer boundary of the coil windings. In this way the magnetic flux density could be concentrated near the sensor without impinging on the area enclosed by the coil windings. Skin depth considerations suggest that a material with high permeability and conductivity will provide the best electromagnetic shielding [2]. The flux amplification associated with permeable materials also helps provide a large flux density at the outer edge of the pickup coil. A ferromagnetic mild steel, 1020 steel, was found to yield good results. The conductivity of the material is large enough to eliminate flux linkage between the coils so that a null signal is measured when testing unflawed material, low conductivity ferrites did not provide adequate shielding to provide this self-nulling feature, and the permeability is high enough to produce the desired flux density at the outer diameter of the pickup coil.

The compact design of the probe requires only minimal instrumentation. Like most eddy

1. Analytical Services \& Materials, 107 Research Drive, Hampton, VA 23666

2. NASA Langley Research Center, Mail stop 231, Hampton, VA 23681

3. Lockheed Engineering \& Sciences Co., 144 Research Drive, Hampton, VA 23666 


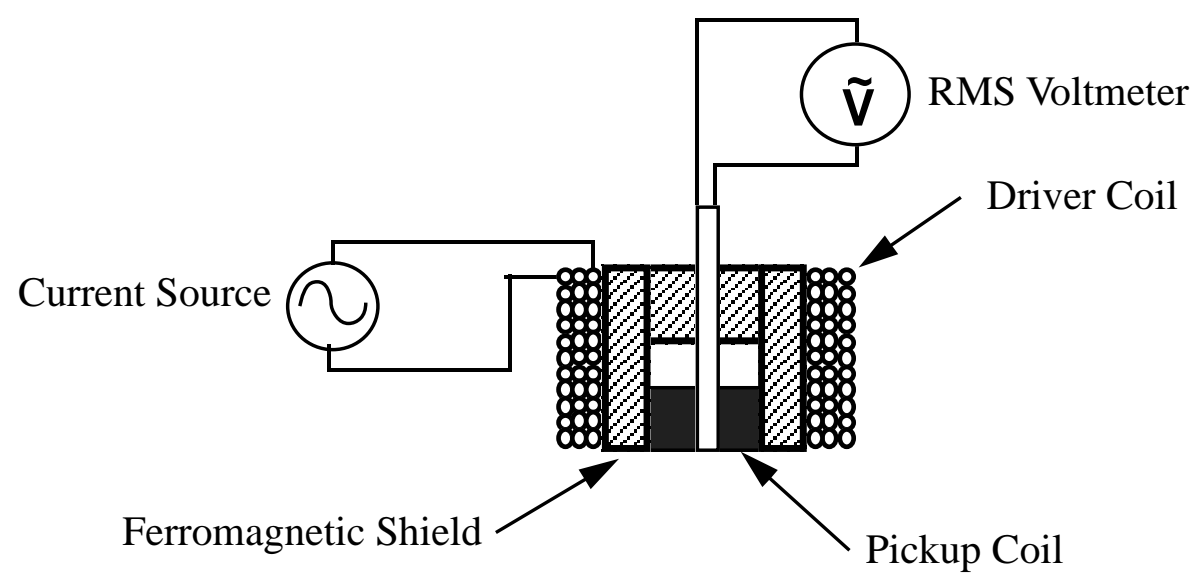

Fig. 1. Schematic diagram of self-nulling eddy current probe.

current techniques, the probe operates with low current input. In addition, the measurement circuitry is less complex than conventional eddy current units, requiring only a voltage measurement circuit. This allows for the construction of a very portable and inexpensive flaw inspection device. Current instruments have been produced operating for extended life times on nine volt batteries with voltage measurements illuminated on LED displays. The combined size of the drive, measurement, and display circuits is approximately $5 \mathrm{~cm}^{2}$. Fig. 2 is a photograph of the portable field unit.

\section{Detection Mechanism for Surface Breaking Flaws}

The self-nulling eddy current probe relies on the magnetic field generated by the eddy currents induced in the sample in order to detect surface flaws. The induced eddy currents will flow to oppose any changes in the magnetic state of the system [4]. Directly under the face of the probe the primary magnetic field is focused by the ferromagnetic lens into a tight cylinder [5]. Consequently, the eddy currents in the sample will be concentrated in a cylindrical region at the outer edge of the pickup coil. Fig. 3 displays finite element modeling results for the eddy current density in an unflawed aluminum plate due to the self-nulling probe. An operating frequency of 70 $\mathrm{kHz}$ was used for the axisymmetric model. The ferromagnetic lens is seen to rapidly attenuate the fields traveling into the lens, shielding the pickup coil from the direct mutual inductance with the drive coil. In the sample, the maximum amplitude of the eddy currents occurs directly under the drive coil windings, decaying rapidly to zero as the pickup coil location is approached. The distribution of the eddy currents, which is directly related to the net magnetic flux in the sample, does not extend far enough under the lens to influence the pickup coil. Thus, the pickup coil is completely shielded from the drive coil field, and a null voltage is recorded.

In the presence of a fatigue crack, the path of the induced current in the sample is distorted. Near the crack tip, current is diverted into the area directly under the pickup coil. The secondary fields due to the eddy currents are then sensed by the pickup coil, and an electromotive force (emf) is generated as determined by Faraday's law of electromagnetic induction [4]. Fig. 4 dis- 
plays the eddy current flow direction in an unflawed sample and as a surface breaking flaw is encountered.

The pickup coil of the self-nulling probe, being a high turn device, develops a large output voltage in response to the small magnetic fields associated with the eddy currents. In addition, the pickup coil voltage, being due only to a distortion in the flow of the eddy currents in the sample, is less sensitive to lift-off and probe wobble than conventional eddy current probes which respond to any changes in the impedance of the system. Far from the sample, some leakage of the primary flux around the ferromagnetic lens occurs and this leakage field interacts with the pickup coil producing a potential difference across the coil leads. The effects of this leakage field for near sample applications can usually be neglected because of the relative amplitudes of the flaw and lift-off signals. Fig. 5 shows the lift-off characteristics of the probe in comparison to the output voltage developed at the tip of a fatigue crack.

\section{Crack Detection Examples}

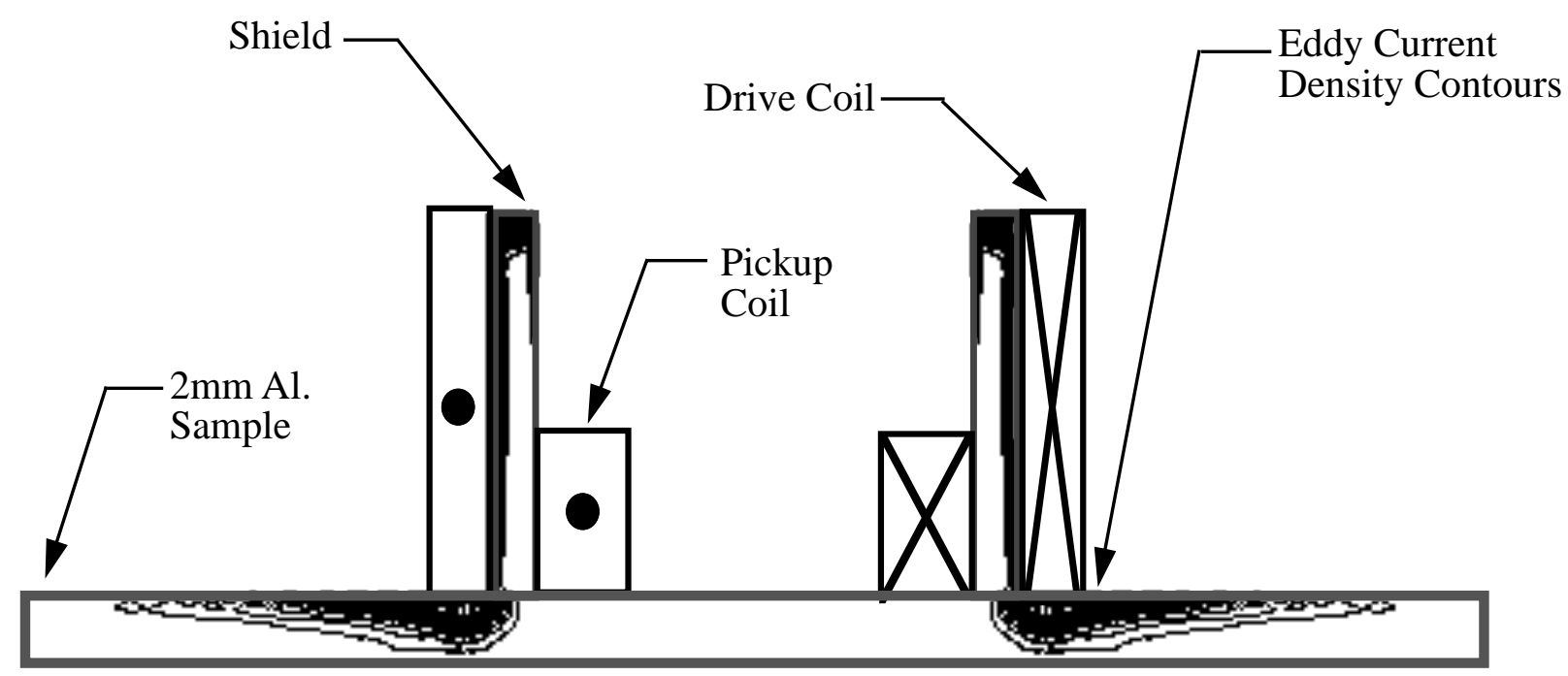

Fig. 3. Finite element results for eddy current density in unflawed sample.

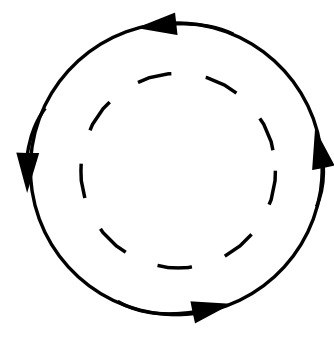

a.

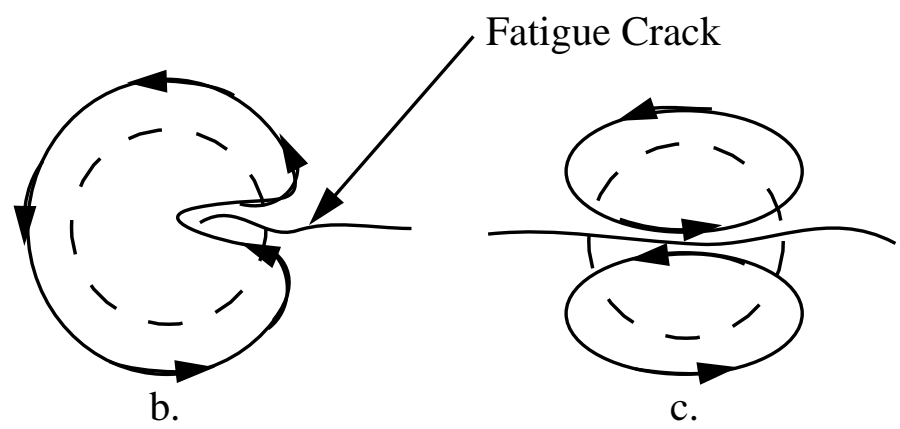

Fig. 4. Eddy current flow of self-nulling probe for (a) unflawed sample, at (b) fatigue crack tip, and (c) at midpoint of crack. Dashed circle gives location of pickup coil. 


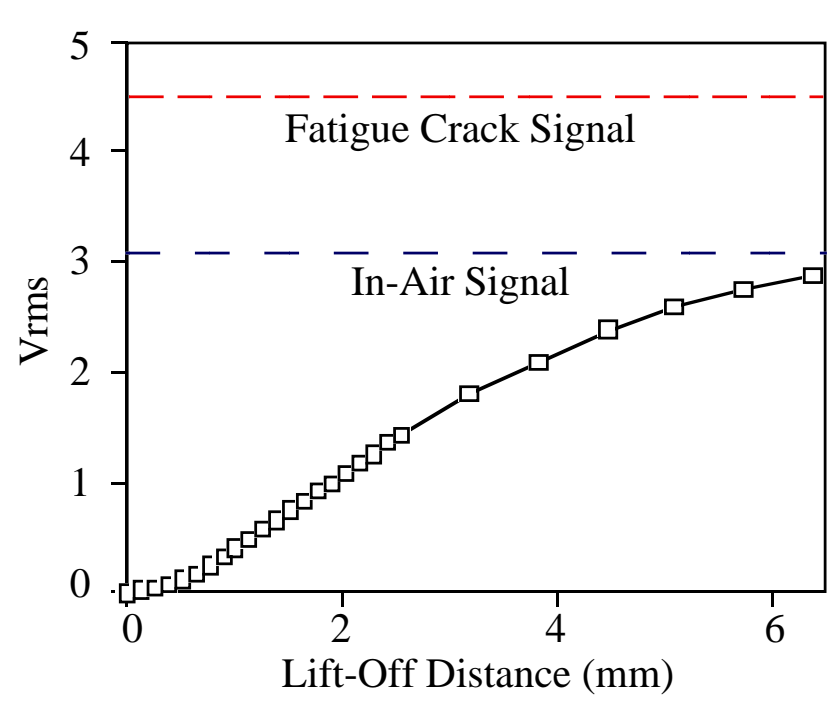

a.

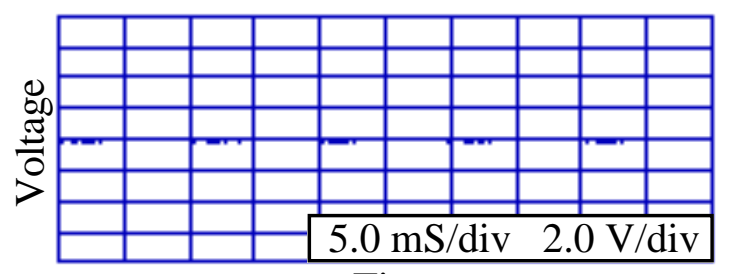

Time

b. Unflawed Material Response

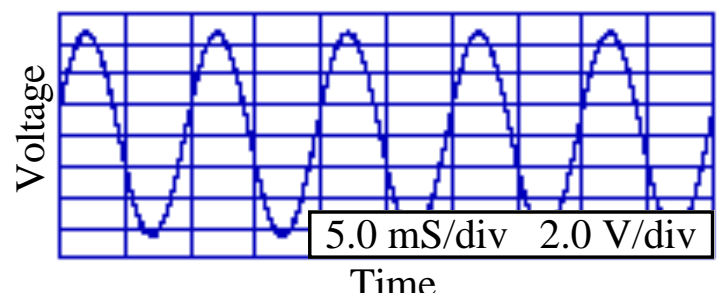

c. Fatigue Crack Response

Fig. 5 Lift-off and fatigue crack signals of self-nulling probe. The raw output signal from the pick-up coil when the probe is placed on unflawed material is shown in b. Only a very small signal level is observed, highlighting the self-nulling property of the probe. Fig. c. displays the pick-up coil output when the probe is located above a fatigue crack. A large amplitude signal is observed at the frequency of the drive signal.

The self-nulling probe has been used to obtain C-scan images of aluminum alloy samples with different types of defects. A raster scan of the samples was performed using an X-Y positioning table while a computer controlled voltmeter recorded the amplitude of the probe output at each $(\mathrm{x}, \mathrm{y})$ position across the sample surface. Three dimensional plots of the data could then be constructed using the probe output voltage as the $\mathrm{z}$ variable.

Fig. 6 and Fig. 7 display C-scan images of EDM notches (19, 12.7, 9, 6, 4, and $2.5 \mathrm{~mm}$ lengths) in a $1 \mathrm{~mm}$ thick aluminum 2024 plate. The figures show results obtained with two different probes. The major difference between the two probes is the inside diameter of the ferromagnetic shield. The data in Fig. 6 was obtained with a probe of $12 \mathrm{~mm}$ ID. This first prototype probe gives an excellent signal for the longer flaws, with a maximum amplitude at the crack tip, as described by Namkung et al [6]. The large diameter of the first prototype probe limits the detectable flaw size of the unit, being unable to isolate the smallest notch. In order to increase the resolution of the device a second probe was constructed and the same notches scanned as in Fig 6. The ID of the second probe was reduced to $6.3 \mathrm{~mm}$. This reduction in probe size is seen to dramatically increase the resolution of the probe, where the $2.5 \mathrm{~mm}$ notch is now detected as displayed in Fig. 7. The minimum detectable flaw size, for isolated flaws, appears to be approximately equal to the radius of the pickup coil. It is anticipated that the resolution of the self-nulling probe can easily be increased 2-3 times with further reductions in probe size.

The characteristics of the self-nulling probe for detecting flaws in riveted structures is displayed in Fig. 8. The figure shows C-scan data obtained with the $6 \mathrm{~mm}$ ID probe. The rivet sample was constructed of $1.6 \mathrm{~mm}$ aluminum 2024 plates, using rivets of $4 \mathrm{~mm}$ shank diameter. EDM notches were machined into the top plate at the location of several rivet holes. The notch lengths varied from $10 \mathrm{~mm}$ down to $1.7 \mathrm{~mm}$ as measured from the rivet shank. The larger diameter probe is seen to detect even the smallest notch, for which only $0.3 \mathrm{~mm}$ extended beyond the edge of the 


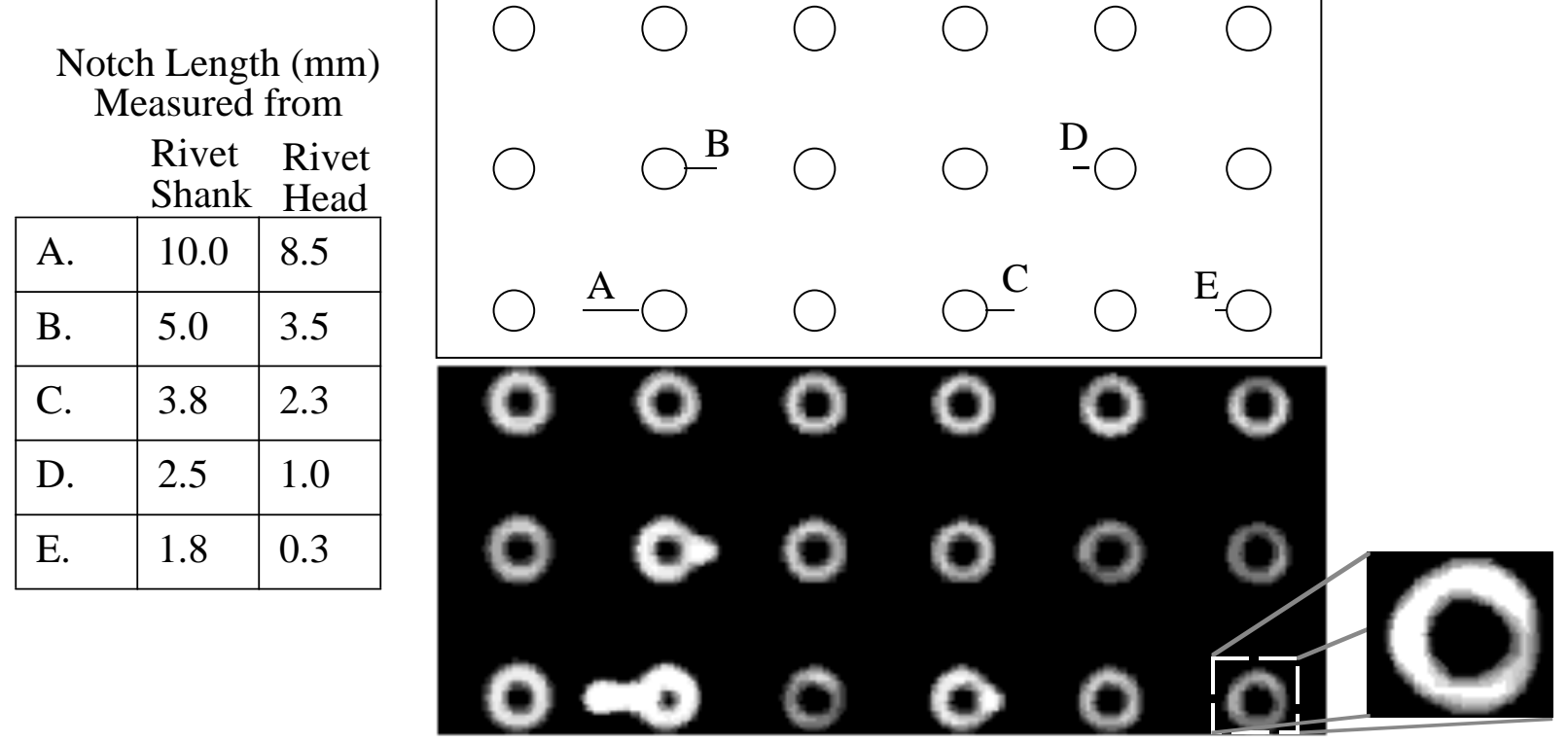

Fig. 8. Detection of EDM notches at rivets with $6 \mathrm{~mm}$ ID self-nulling probe. Notch Lengths are as measured from rivet shank and length protruding past rivet head.

rivet head. Reduction in probe size is expected to more clearly isolate small flaws at rivet heads.

\section{Corrosion Detection}

The operational characteristics of the self-nulling probe change as the frequency is lowered. Under low frequency operation, a significant portion of the magnetic flux of the drive coil is able to penetrate deep into the sample. As the fields travel into the sample they are able to circle around the bottom of the shield and interact with the pickup coil [7]. The probe no longer gives a null output voltage when in contact with unflawed material, but yields an output voltage directly related to the thickness of the sample under test [7]. Material thickness gauging and corrosion detection can therefore be accomplished by operating the probe at a low frequency and monitoring the output voltage.

The corrosion detection capabilities of the self-nulling probe are illustrated in Fig. 9. A simulated interlayer corrosion sample was constructed by removing material from the back surface of a $1.6 \mathrm{~mm}$ aluminum alloy plate. The sample was then bonded to another aluminum plate, sandwiching the machined surface between the plates. The amount of material loss was varied from 
1.5 - $6 \%$ of the base thickness along an $\mathrm{N}$-shaped path in the center of the sample. The C-scan data displayed in Fig. 9 easily identifies the material loss region, having excellent agreement with the actual measured values as recorded by mechanical measurements performed before the two plates were bonded together.

\section{Summary}

A new eddy current probe has been developed in order to simplify non-destructive testing and reduce inspection times without sacrificing flaw resolution. The self-nulling eddy current probe requires no calibration, reference standards or balance circuitry in order detect surface breaking flaws with a high probability of success. A zero, or null voltage is displayed when an unflawed material is inspected. The presence of flaws in the material eliminates the nulling condition and a large output voltage is recorded, peaking at the tip of the flaw.

The probe has been shown to detect isolated flaws down to $2.5 \mathrm{~mm}$, and reductions in probe size are expected to reduce this value below $1 \mathrm{~mm}$. In riveted structures the resolution is shown to be even greater as small distortions in the current path at the rivet/base metal interface produce measurable changes in the output voltage of the probe. The detection of a notch protruding less than $0.5 \mathrm{~mm}$ beyond the rivet head has been illustrated. The capabilities of the probe at detecting corrosion damage have also been introduced, where low frequency operation, although destroying the self-nulling properties of the probe, can yield valuable information of the thickness of the part being tested.

The simple instrumentation of the self-nulling probe has lead to the production of prototype field units using nine volt batteries as a power source and having a total weight of less than 0.25 $\mathrm{kg}$. Commercialization of the device should lead to an inexpensive, portable, NDT instrument for surface and subsurface flaw detection.

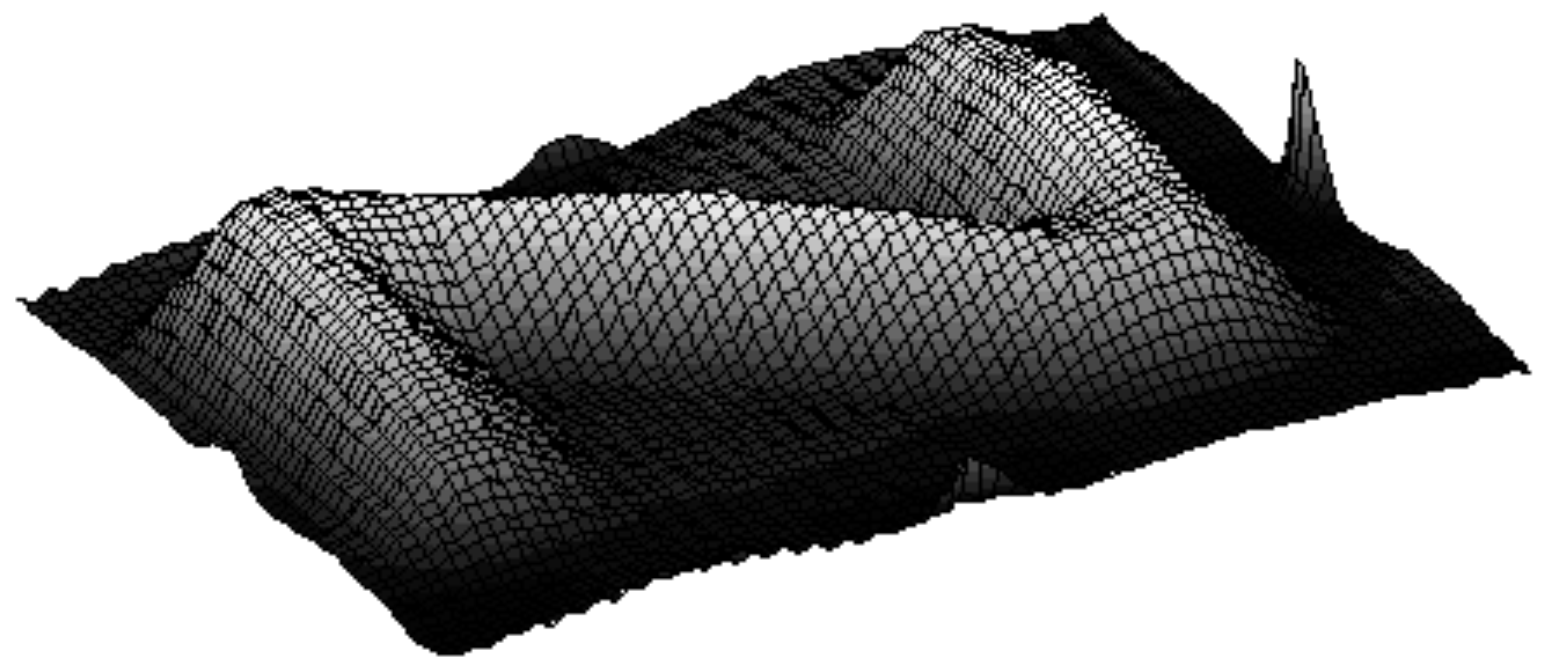

Fig. 9. Detection of simulated interlayer corrosion damage with self-nulling probe. 


\section{References}

1. J.W. Simpson, B. Wincheski, M. Namkung, J.P. Fulton, R.G. Todhunter, and C.G. Clendenin, "Flux Focusing Eddy Current Probe and Method for Flaw Detection," Patent Pending.

2. Libby, H.L., Introduction to Electromagnetic Nondestructive Test Methods, John Wiley \& Sons, Inc., New York, 1971, Chapter 5.

3. Nondestructive Testing Handbook, Volume Four, edited by Paul McIntire, American Society for Nondestructive Testing, Inc., 1986, Section 1, Part 3.

4. Jackson, J.D., Classical Electrodynamics, Second Edition, John Wiley \& Sons, Inc., New York, 1975, Chapter 6.

5. B. Wincheski, J.P. Fulton, M. Namkung, J.W. Simpson, and S. Nath, "Characteristics of Ferromagnetic Flux Focusing Lens in the Development of Surface/Subsurface Flaw Detection, "Presented at 1993 Review of Progress in QNDE, Brunswick ME., August 1-6, 1993, Proceedings to be published by Plenum Press, New York.

6. M. Namkung, C.G. Clendenin, J.P. Fulton, and B. Wincheski, "An Application of a New Electromagnetic Sensor to Real-Time Monitoring of Fatigue Crack Growth in Thin Metal Plates, ibid.

7. B. Wincheski, M. Namkung, J.P. Fulton, and S. Nath, "New Eddy Current Device for Thickness Gauging of Conductive Materials," ibid. 